\title{
The Loss of Correction in Thoracolumbar Burst Fracture Treated by Surgery. Can We Predict It?
}

\author{
Anghel S1,2, Márton D1,2 \\ PhD Student, University of Medicine and Pharmacy, Tîrgu Mureș, Romania \\ 2 Clinic of Orthopedics, County Emergency Clinical Hospital, Tîrgu Mureș, Romania
}

Objective: This paper aims to differentially depict potential patterns of the loss of correction in surgically treated thoraco-lumbar burst fractures. These may eventually serve to foreseeing and even forestalling loss of correction.

Methods: The study focused on 253 patients with surgically treated thoraco-lumbar fractures. This cohort of patients was clustered in four subgroups according to the fracture spine segment (T11-L1 or L1-L2) and surgery type (short segment fixation or anterior approach). Relevant recorded and processed data were the fracture level, post-operative (Kpo) and last follow-up (Kf) kyphosis angle values. Correlation, regression and determination testing were performed for the last follow-up kyphosis angle and post-operative kyphosis angle, and regression equations were determined for each subgroup of patients.

Results: The patterns of loss of correction were described through the following equations: $K_{f}=0.95^{\star} K_{p o}+3.2^{\circ}$ for the $T 11-L 1$ level fractured vertebrae treated by posterior short segment fixation; $K_{f}=0.98^{*} K_{p o}+3.4^{\circ}$ for the $L 1-L 2$ level fractured vertebrae treated by posterior short segment fixation; $K_{f}=1.1^{\star} K_{p o}+1.6^{\circ}$ for the $T 11-L 1$ level fractured vertebrae treated by anterior approach; and $K_{f}=0.7^{\star} K_{p o}+2.8^{\circ}$ for the L1-L2 level fracture vertebrae treated by anterior approach.

Conclusions: The loss of correction may be predicted, to a certain extent, for thoraco-lumbar fractured vertebrae treated surgically. The bestfit equations depicted for both type of surgery (short segment fixation and anterior approach) and both spinal segments (T11-L1 and L2-L3) are significantly different than the equations delineated for the collapse of non-surgically treated fractures.

Keywords: burst fracture, thoracolumbar spine, kyphosis, loss of correction

Received: 30 April 2013 / Accepted: 11 May 2014

\section{Introduction}

The thoracolumbar burst fracture in neurologically intact patients may be treated either conservatively or surgically. It is generally agreed that stable fractures should be orthopedically attended, while the unstable ones should be treated by surgery. There is, however, much debate on the proper definition and classification of fracture stability [1, 2] and the proper treatment for burst fracture according to the specific stability level [3].

Surgically treated thoracolumbar burst fracture is often followed by significant loss of correction which impairs the daily activities performance, induces pain and may even require another operative correction. As a result of the fracture, the vertebral body experiences a certain degree of vertebral wedging, causing some angulation of the spinal segment. The surgical procedure aims at restoring the normal spinal angulation through recovering the height of the vertebral body and its fixation for the fracture healing. The conservative treatment, on the other hand, is intended to only ensure the proper healing conditions. Orthopedic maneuvers for fracture reduction are also occasionally performed.

Regardless of the treatment type applied, it has been noticed that the local kyphosis angle tends to get narrower [3-6]. Sometimes the angulation gets so significant that it

Correspondence to: Stelian Anghel

E-mail: steliananghel@yahoo.com brings forth important sagittal plane imbalance, together with pain, and sometimes even neurological deficit [7]. This complication is called post-traumatic kyphosis, that is surgically treated in order to restore spinal balance. This intervention is much more complex than the initial fracture treatment, displaying higher morbidity rates than the case of effective early surgical treatment. Vaccaro et al. [8] include the ineffective treatment of the fracture among the main causes of the post-traumatic kyphosis. This may imply that, for some reason, the deformation potential of the fracture hadn't been fully estimated at the beginning.

In their work, Fahad K. Al-Khalifa et al. [9] determined specific best-fit regression equations for the collapse pattern in the case of conservatively treated burst fractures, correlating the initial and post-treatment kyphosis angles. Thus, they depicted the equation $K_{f}=K_{i}+0.5 K_{i}$ for burst fracture at T11-L1 level and the equation $K_{f}=K_{i}+4$ for the burst fracture at $\mathrm{L} 2-\mathrm{L} 3$ level (where $\mathrm{K}_{\mathrm{i}}$ is the kyphosis angle measured at the time of admission and $K_{f}$ is the kyphosis angle at the last follow up, which was at least one year from the trauma).

Hereafter, this research paper aims to study if the same formulae may apply to the loss of correction that appears after a surgically treated thoracolumbar burst fracture.

\section{Methods}

The relevant literature was selected through a systematic review of the research papers published between 2000 and 
2012, and available in the databases Sciencedirect, Ovid and PubMed, using "burst fracture" as search parameter. The inclusion criteria were the following:

- to involve thoracal and/or lumbar fractures;

- all cases to be only traumatic spine fractures, not related to osteoporosis or any other pathology;

- the last follow-up exceeds one year;

- detailed and specific information regarding all important data on each patient: demographic data (sex and age), injury data (spine level, post-traumatic kyphosis angle), treatment (type of the surgery) and follow-up data (time, final kyphosis angle).

The 10 articles - out of 33 - that met the criteria and were included in the final database, discuss the most frequently employed surgical treatment methods for the thoracal and lumbar fractures: posterior short segment fixation [10-15], on one hand, and anterior approach only, as well as posterior-anterior approach [16-19], on the other.

Of all cases presented, we considered only the patients that satisfied the following minimal requirements:

- only one level was affected;

- the affected vertebra was mentioned;

- the pre-operative, post-surgical and last follow-up kyphosis angles were given.

The lot was clustered after the type of surgical intervention type - posterior short segment fixation or anterior/ anterior-posterior approach. Each group was further fractioned in two subgroups, according to the affected spinal level: the subgroup of the thoraco-lumbar junction fractures (T11-L1) and the subgroup of the L2-L3 level fractures. We left out another potential subgroup of L4-L5 level fractures, due to insufficient data available.

The extracted and recorded data consists of post-operative $\left(\mathrm{K}_{\mathrm{po}}\right)$ and last follow-up $\left(\mathrm{K}_{\mathrm{f}}\right)$ kyphosis angles for each entity. This information was then statistically analysed through correlation, linear regression and determination between the two variables $\left(\mathrm{K}_{\mathrm{po}}\right.$ and $\left.\mathrm{K}_{\mathrm{f}}\right)$ and a best-fit equation was delineated within each subgroup of surgical approach. The statistical software employed was GraphPad Instat.

\section{Results}

The selected papers provided information for 253 patients with surgically treated thoraco-lumbar fractures, out of which 177 were treated with posterior short segment fixation and 76 by anterior approach.

The subgroup with thoraco-lumbar junction fractures (T11-L1) of the posterior short segment fixation group comprised 96 patients. We determined the corresponding best fit correlation equation as being $\mathrm{K}_{\mathrm{f}}=0.95^{*} \mathrm{~K}_{\mathrm{po}}+$ $3.2^{\circ}$, where $\mathrm{K}_{\mathrm{f}}$ is the last follow-up measured kyphosis angle and $\mathrm{K}_{\mathrm{po}}$ is the post-operative kyphosis angle. Also, for this subgroup we recorded good values of statistical significance $(\mathrm{p}=0.0001, \mathrm{r}=0.84$ and $\mathrm{r} 2=0.72)$ (Figure 1a). For the subgroup with L2-L3 level fractures of the posterior short segment fixation group, consisting of 75 patients, the determined correlation equation was $\mathrm{K}_{\mathrm{f}}=0.98^{*} \mathrm{~K}_{\mathrm{po}}+3.4^{\circ}(\mathrm{p}=$

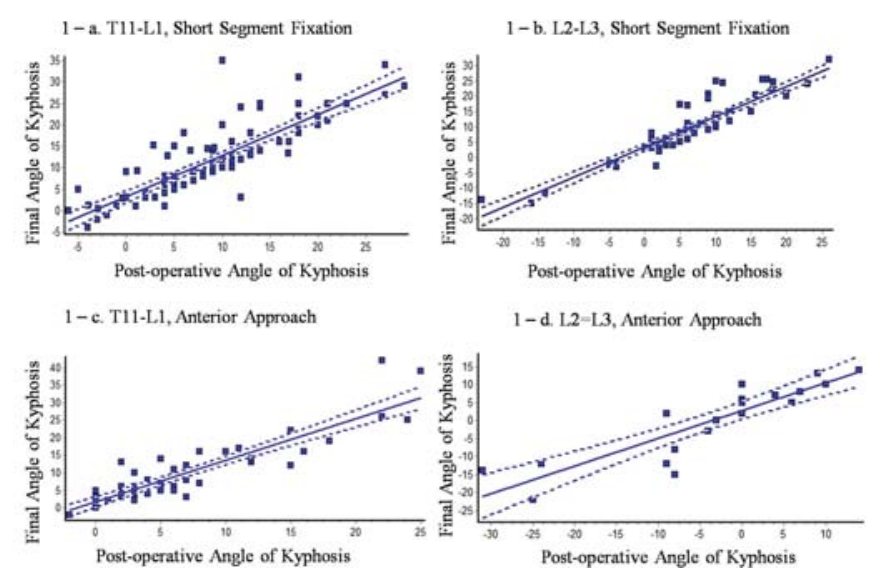

Fig. 1. Regression lines for each subgroup of patients

$0.0001, \mathrm{r}=0.92$ and $\mathrm{r} 2=0.85$ ) (Figure $1 \mathrm{~b}$ ). Only 6 patients presented L4-L 5 level fractures and this subgroup was eliminated from the study as non-relevant, given its small size.

Fifty-four patients with fractures at T11-L1 level were treated by anterior approach. After statistically processing data, the emergent equation was $\mathrm{K}_{\mathrm{f}}=1.1^{*} \mathrm{~K}_{\mathrm{po}}+1.6^{\circ} ; \mathrm{p}=$ $0.0001, \mathrm{r}=0.9$ and $\mathrm{r} 2=0.81$ (Figure 1c). For the subgroup of 21 patients with L2-L3 level fractures that were treated with anterior approach type surgery, the resulting best fit correlation equation is $\mathrm{K}_{\mathrm{f}}=0.7^{*} \mathrm{~K}_{\mathrm{po}}+2.8^{\circ}(\mathrm{p}=0.0001, \mathrm{r}=$ 0.86 and $\mathrm{r} 2=0.78$ ) (Figure $1 \mathrm{~d}$ ). One patient presented a L4 level fracture and associated data was removed from the study, as non-relevant.

\section{Discussion}

Earlier research papers have determined that anatomically distinct spinal segments denote different specific biomechanical behaviors and have identified different collapse patterns of the vertebral body for conservatively treated burst fractures in various spine areas [9].

Given the anatomic specific characteristics of the spine, with physiological curvatures, the compressive forces differ from one vertebra to another. The length of the force arm depends on the particular position of the vertebral body center against the plumbline, and on the human body's center of mass. The farther the vertebral body center from the plumbline, the longer the force arm is and, consequently, the higher compression load on the vertebral body. Therefore, we may infer that the magnitude of the forces would be higher in kyphotic segments and lower in lordotic spine.

At the same time, each vertebral body sustains the segment of the human body above it. Therefore, the axial force increases as the level of the vertebra is lower positioned.

Moreover, Panjabi [20] argue that, in case of a fractured and angled vertebra, the force arm increases proportionally with the wedge angle.

As we have mentioned before, Fahad K. Al-Khalifa et al. [9] found that the collapse of a fractured vertebra treated non-surgically may be to a certain extent predicted, applying the equation $\mathrm{K}_{\mathrm{f}}=\mathrm{K}_{\mathrm{i}}+0.5 * \mathrm{~K}_{\mathrm{i}}$ for a T11-L1 level frac- 
tured vertebra, and $\mathrm{K}_{\mathrm{f}}=\mathrm{K}_{\mathrm{i}}+4^{\circ}$ for a L2-L3 level fractured vertebra. For example, given a fracture displaying an initial kyphotic angle of $20^{\circ}$ - which is considered by some authors as surgery treatment indication [21, 22], for a T11-L1 segment position, according to the equation, the kyphotic angle would increase up to $30^{\circ}$. This would require, as well, surgical intervention for post-traumatic kyphosis treatment [8]. On the other hand, if the injured vertebra were situated in L2-L3 segment, the final angle would be of $24^{\circ}$.

In our study, as a specific contribution of this paper, we have also identified equations for the loss of correction for each subgroup of surgically treated fractured vertebrae. These formulae are considerably different from those associated with non-surgically treated fractured vertebrae, cited above [9]. At the same time, it is apparent that the variation between the different spinal segments for the same surgery type is smaller. This may suggest that, the surgical treatment itself reduces the variation between the impact of the different forces stressing the two segments, which is significant in case of non-surgically treated fractures.

For a surgically treated fractured vertebra, the final kyphosis angle is determined by the post-operative kyphosis angle and a constant specific to each vertebral segment, which, however, does not differ widely between segments for the same operation type. All subgroups present a very strong correlation between the degree of loss of correction and the post-operative kyphosis angle, which has been expected.

The postoperative correction level is largely dependent on the human factor. However, given that the final kyphosis angle is also determined by a constant, an over-correction of the fracture by the size of the constant might lead to a final kyphosis angle closer to the normal physiological values.

At the same time, it is worth noting that, for the group of patients that were treated by short segment fixation, there is considerable difference between R-square coefficients related to the two spinal segments. This implicates that the probability for a particular case fracture treated by short segment fixation not to fit in the correspondent equation is higher for T11-L1 level fractured vertebrae than L2L3 level. In other words, there is a $28 \%$ probability for a fracture at T11-L2 level (the highest probability among all subgroups) to develop a greater loss of correction than predicted by correspondent equation. This segment has been considered more vulnerable to post-traumatic kyphosis by other authors as well [7].

The main limitation of our research lays in the lack of information regarding the weight of the patients, which may represent a relevant independent variable.

\section{Conclusions}

To a certain extent, the loss of correction may be predicted for thoraco-lumbar fractured vertebrae treated surgically. Generally speaking, there are about $80 \%$ chances that the outlined equations predict the development of the loss of correction, except for the fractures positioned in T11-L1 segment, treated by short segment fixation.
The best-fit equations depicted for both type of surgery (short segment fixation and anterior approach) and both spinal segments (T11-L1 and L2-L3) are significantly different than the equations delineated for the collapse of non-surgically treated fractures.

\section{Acknowledgement}

This paper is partly supported by the Sectorial Operational Programme Human Resources Development (SOP HRD), financed from the European Social Fund and by the Romanian Government under the contract number POSDRU 80641.

\section{References}

1. White AA JR, Panjabi MM, et al. Biomechanical analysis of clinical stability in the cervical spine. Clinical Orthopaedics and Related Research. 1975;109:85-96.

2. Kirkaldy-Willis WH. Presidential symposium on Instability of the Lumbar Spine. Introduction. Spine. 1985;10:254

3. Dai LY, Jiang LS, Jiang SD. Conservative Treatment of Thoracolumbar Burst Fractures. Spine. 2008;33(23):2536-2544.

4. Chow GH, Nelson BJ, Gebhard JS, et al. Functional outcome of thoracolumbar burst fractures managed with hyperextension casting or bracing and early mobilization. Spine. 1996;21:2170-2175.

5. Tropiano P, Huang $\mathrm{RC}$, Louis $\mathrm{CA}$, et al. Functional and radiographic outcome of thoracolumbar and lumbar burst fractures managed by closed orthopaedic reduction and casting. Spine. 2003;28:2459-2465.

6. McLain RF SE, Benson DR. Early failure of short-segment pedicle instrumentation for thoracolumbar fractures. A preliminary report. J Bone Joint Surg Am. 1993;75:162-116.

7. Schoenfeld AJ, Wood KB, Fisher CF, et al. Posttraumatic Kyphosis: Current State of Diagnosis and Treatment: Results of a Multinational Survey of Spine Trauma Surgeons. J Spinal Disord Tech. 2010;23(7):e1-8.

8. Alexander R, Vaccaro M, Silber JS. Post-traumatic Spinal Deformity. Spine. 2001;26(24S):S111-S118.

9. Al-Khalifa FK, Adjei N, Yee AJ, Finkelstein JA. Patterns of Collapse in Thoracolumbar Burst Fractures. J Spinal Disord Tech. 2005;18:410-412.

10. Liu YJ, Wang ST, Yu WK, Liu CL, Chen TH. Flexion-distraction injury of the thoracolumbar spine. Injury, Int J Care Injured. 2003;34:920-923.

11. Helton LAD, Canto FRT. Low thoracic and lumbar burst fractures: radiographic and functional outcomes. Eur Spine J. 2007;16:1934-1943.

12. Ko SB, Lee SW, Baek SH, et al. Result of Posterior Instrumentation Without Fusion in the Management of Thoracolumbar and Lumbar Unstable Burst Fracture. Journal of Spinal Disorders \& Techniques. 2014;(27(4):189-95.

13. Parker JW, Lane JR, Karaikovic EE, Gaines RW. Successful ShortSegment Instrumentation and Fusion for Thoracolumbar Spine Fractures. Spine. 2000;25(9):1157-1169.

14. Gelb D, Ludwig S, Karp JE, et al. Successful Treatment of Thoracolumbar Fractures With Short-segment Pedicle Instrumentation. J Spinal Disord Tech. 2010;23(5):293-301

15. Toyone T, Tanaka T, Kato D, Kaneyama R, Otsuka M. The Treatment of Acute Thoracolumbar Burst Fractures With Transpedicular Intracorporeal Hydroxyapatite Grafting Following Indirect Reduction and Pedicle Screw Fixation - A Prospective Study. Spine. 2006;31(7):E208-E214.

16. Suzuky T, Miyakoshi N, Murai H, et al. Anterior Decompression and Shortening Reconstruction With a Titanium Mesh Cage Through a Posterior Alone for the Treatment of Lumbar Burst Fractures. Asian Spine Journal. 2012;6(2):123-130.

17. Wood KB, Bohn D, Mehbod A. Anterior Versus Posterior Treatment of Stable Thoracolumbar Burst Fractures Without Neurologic Deficit. J Spinal Disord Tech. 2005;18(suppl 1):S15-S23.

18. Sasani M, Ozer AF. Single-Stage Posterior Corpectomy and Expandable Cage Placement for Treatment of Thoracic or Lumbar Burst Fractures. Spine. 2008;34(1):E33-E40.

19. Haiyun Y, Rui G, Shucai D, et al. Three-Column Reconstruction Through Single Posterior Approach for the Treatment of Unstable Thoracolumbar Fracture. Spine. 2010;35(8):E295-E302.

20. Panjabi M, Monohar AAWI. Cinical Biomechanics of the Spine, second ed. Philadelphia: J.B. Lippincott Company; 1990.

21. Benson DR, Montesano PX, Sutherland TB, McLain RF. Unstable thoracolumbar and lumbar burst fractures treated with the AO fixateur interne. J Spinal Disord Tech. 1992;5:335-343.

22. Willen JAJ, Toomoka K, Singer K. The natural history of burst fractures at the thoracolumbar junction. J Spinal Disord. 1990;3:39-46. 